http://dx.doi.org/10.18778/0208-6107.32.09

\author{
Damir Barbarić \\ Institut für Philosophie \\ Universität Zagreb \\ barba@ifzg.hr
}

\title{
PATHEI MATHOS. SCHOPENHAUERS WEISHEITSLEHRE UND DIE ALTGRIECHISCHE SOPHIA?
}

\begin{abstract}
Zusammenfassung
Im Angesicht der wachsenden Entsubjektivierung und Depersonalisierung, die sich im Bereich der Erkenntnis und des Wissens durch den unbedingten Anspruch der Wissenschaft auf die Objektivität durchgesetzt haben, empfiehlt es sich, an den alten, neuerdings größtenteils zur Vergessenheit gesunkenen Begriff der Weisheit zu erinnern und ihn seiner philosophischen Tragweite nach zu prüfen. Hier wird das am Beispiel Schopenhauer gemacht. In seiner Philosophie spielt die Weisheit eine ganz zentrale Rolle, und zwar als eine nicht bloß theoretische, sondern auch praktische Vollkommenheit des Menschen. Um das Wesen der so hoch gesetzten Weisheit möglichst genau zu fassen, wird sie im Aufsatz zum einen mit Gadamers Begriff der hermeneutischen Erfahrung und zum anderen mit dem in altgriechischem Spruch „,durch Leid gelehrt" zum Ausdruck kommenden Weisheitsbegriff konfrontiert. Die Untersuchung führt zum Ergebnis, dass die Weisheit bei Schopenhauer, obwohl auf den ersten Blick den beiden erwähnten Weisheitsauffassungen ähnlich und teilweise sogar verwandt, von ihnen jedoch von Grund auf verschieden ist, da ihr Zweck nicht darin besteht, den Menschen über die unüberwindliche Grenzen seiner Sterblichkeit zu belehren und damit ihm seinen zugemessenen Platz im Leben zuzuweisen, sondern vielmehr ihm von dem Willen zum Leben überhaupt wegzuführen.
\end{abstract}

\section{Schlüsselwörter:}

Schopenhauer, Weisheit, Wille, Erkenntnis, Schmerz, Leiden

Von verschiedenen Seiten werden in der neueren Zeit das wissenschaftliche Wissen und seine Funktion im Ganzen der Lebenswelt in Frage gestellt und einer tiefgreifenden Kritik unterworfen. Um ein Beispiel zu geben, sei hier auf die vor zehn Jahren vorgebrachte m. E. treffende Diagnose von Karen Gloy hingewiesen: 
Der Typ des wissenschaftlichen Wissens, der aufgrund seiner Formalisierbarkeit, Quantifizierbarkeit und Logifizierbarkeit objektivierbar ist, in Büchern, auf Chips, in Datenbanken gespeichert werden kann und daher jederzeit abrufbar ist, hat es mit sich gebracht, daß er mit der Entbindung aus der persönlichen Erfahrung des Subjekts auch aus der ethischen Bindung entlassen wurde. ${ }^{1}$

Gloy plädiert dafür, solche die Lebenswelt gefährdende Tendenzen der immer weiter schreitenden ethisch ungebundenen Wissenschaft dem breiteren Rahmen einer ihr die Grenzen zeigenden Weisheit wieder einzufügen, jener nämlich, deren Begriff mit der neuzeitlichen „Entsubjektivierung und Depersonalisierung, mit dieser Verobjektivierung der Wissenschaften obsolet" wurde. $^{2}$ Denn die „Grundbedingung“ der Weisheit als „ethisch orientierten Lebensführung $^{\text {“3 }}$, wie sie etwa unter dem griechischen Namen sophia und dem lateinischen sapientia sowie den deutschen Namen Witz, Kunst und List im späten Mittelalter und frühen Neuzeit verstanden wurde, war die ,noch ungebrochene Einheit von

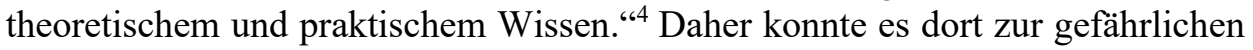
Verselbstständigung der vom Ganzen der Lebenswelt völlig entbundenen und abgeschnittenen, im Grunde anonym verlaufenden bloß theoretischen Wissenschaft nicht kommen. Heute gilt es auf diesen wesentlichen Unterschied zu erinnern. Denn

[e]s ist etwas anderes ob das Wissen in persönlicher Lebenserfahrung gründet, ein im individuellen Existenzkampf gewonnenes, dem Leben abgerungenes Wissen ist, das sowohl die eigenen Fähigkeiten wie auch die eigenen Grenzen erkennen läßt, oder ein vom Individuum und seiner Erfahrung abgespaltenes, allgemeines, intersubjektiv kommunikables Wissen, das aufgrund der Objektivation wertneutral ist und aus der unmittelbaren Bewertung und Verantwortung des Einzelnen entlassen wurde. ${ }^{5}$

Gewiss reicht die Erinnerung an das Ehemalige nicht dazu, die Laufbahn der Geschichte, die seit Jahrhunderten durch die verabsolutierte Wissenschaft geleitet wird, von Grund auf in Frage zu stellen, geschweige sie zu verändern. Um die Logik und die Genese dieser Geschichtslauf durchzuschauen und zur begrifflichen Erkenntnis zu bringen, ist es viel mehr erforderlich. In vollem Bewusstsein dieser Einschränkung möchte ich hier eine Weisheitskonzeption in ihren Grundzügen darstellen, die in unserem Zusammenhang sicher nicht belanglos ist, nämlich jene Schopenhauers.

\footnotetext{
${ }^{1}$ Karen Gloy, Von der Weisheit zur Wissenschaft (Freiburg/München: Verlag Karl Alber, 2007), 102.

${ }^{2}$ Ibid., 37.

${ }^{3}$ Ibid., 41.

${ }^{4}$ Ibid., 80.

${ }^{5}$ Ibid., 103.
} 
Jede wissenschaftliche Erkenntnis, jene der positiven Wissenschaften wie auch jene der wissenschaftlich verfahrenden Naturphilosophie, spielt sich in einem Bereich ab, der durchgängig vom Satz von dem zureichenden Grunde bestimmt ist. Alles, was in diesem Bereich begegnet, ist der zeitlichen Sukzession sowie dem räumlichen Nebeneinander, daher auch der Zahl und der Größe sowie dem allgemeinen Gesetz der Kausalität unterworfen. Um den Schritt hinauf über diesen Bereich, der dem Menschen unmittelbar gegeben ist und ihm zunächst als das Einzige, was es gibt, erscheint, zu machen, tut es Not, mit Kant den transzendentalen Abstand vor diesem Bereich zu nehmen und ihm zwar die empirische Realität, aber nichtsdestoweniger die transzendentale Idealität zuzumessen. Eben das tut Schopenhauer:

Insofern ist also die angeschaute Welt in Raum und Zeit, welche sich als lauter Kausalität kundgibt, vollkommen real und ist durchaus das, wofür sie sich gibt, und sie gibt sich ganz und ohne Rückhalt als Vorstellung, zusammenhängend nach dem Gesetz der Kausalität. Dieses ist ihre empirische Realität. Andererseits ist aber alle Kausalität nur im Verstande und für den Verstand; jene ganz wirkliche, d. i. wirkende Welt, ist also als solche immer durch den Verstand bedingt und ohne ihn nichts. [...] Die ganze Welt der Objekte ist und bleibt Vorstellung und eben deswegen durchaus und in alle Ewigkeit durch das Subjekt bedingt: d. h. sie hat transzendentale Idealität. ${ }^{6}$

Vom transzendentalen Gesichtspunkt zeigt sich also das Ganze der unter dem Satz des zureichenden Grundes stehenden Dinge, dem irreführenden, obwohl notwendigen Schein ihrer Realität entgegen, nur als ein Bereich bloßer Vorstellungen, und d. h. Erscheinungen:

Alles Objektive, Ausgedehnte, Wirkende, also alles Materielle [...] ist ein nur höchst mittelbar und bedingterweise Gegebenes, demnach nur relativ Vorhandenes: denn es ist durchgegangen durch die Maschinerie und Fabrikation des Gehirns und also eingegangen in deren Formen, Zeit, Raum und Kausalität, vermöge welcher allererst es sich darstellt als ausgedehnt im Raum und wirkend in der Zeit. ${ }^{7}$

Die einzige Art des Erkennens innerhalb dieses Bereichs der Vorstellungen bzw. Erscheinungen ist entweder die durch den Verstand vermittelte und geleitete Sinneswahrnehmung der Einzelerscheinungen oder der abstrakte, diskursive Begriff, der deren mehrere Vorstellungen unter sich mechanisch vereinigt. Wie ihr Gegenstand ist auch diese Erkenntnis stets nur relativ und ins Unendliche fortschreitend. Jegliches darin Erkannte wird gefasst immer nur in Hinsicht auf etwas anderes, nie aber als solches und an sich. Da die gegenseitigen Verhältnis-

\footnotetext{
${ }^{6}$ Arthur Schopenhauer, „Die Welt als Wille und Vorstellung I,“ in Arthur Schopenhauer, Sämtliche Werke, textkritisch bearbeitet und herausgegeben von Wolfgang Frhr. von Löhneysen (Frankfurt am Main: Wissenschaftliche Buchgesellschaft Darmstadt, 1968), $45 \mathrm{f}$.

${ }^{7}$ Schopenhauer, „Die Welt als Wille und Vorstellung I,“ 63.
} 
se der Einzelerscheinungen ihrer Zahl nach unbegrenzt sind, schreitet solche Erkenntnis unaufhörlich auf deren Spur immer fort, hält sich nie auf und steht bei keinem Erkannten stille. Wissenschaftliches Erkennen ist also zwar endlos, kommt aber nie zur wahren Erkenntnis eines Dinges, wie es an sich ist.

Solches gelinge erst dann, wenn das Erkennen den Gang der unaufhörlich fortschreitenden und am Leitfaden der Kausalität und des Satzes vom Grunde sich bewegenden Forschung verlässt und sich damit selbst dem endlosen Strom der Erscheinungen entreißt. Das einzig geeignete Mittel dazu ist die Anschauung. ${ }^{8}$ Zum Unterschied vom begreifenden Verfahren geläufiger Wissenschaft, die an einer Einzelerscheinung stets ihre Verhältnisse zu die anderen verfolgt und sich insofern bei ihr selbst eigentlich nie aufhält, geht die intuitive Anschauung „nur auf das Nächste und bleibt bei diesem stehn.“9 So erlangt die Erkenntnis erst durch die Anschauung und in der Anschauung die sonst immer gesuchte, aber in der diskursiv verfahrenden Wissenschaft nie zu erreichende Ruhe und den festen Besitz der Gegenwart: „Solange wir uns rein anschauend verhalten, ist alles klar, fest und gewiß. Da gibt es weder Fragen noch Zweifeln noch Irren: man will nicht weiter, kann nicht weiter, hat Ruhe im Anschauen, Befriedigung in der Gegenwart." ${ }^{\text {10 }}$

Was in der Anschauung eigentlich erkannt wird, ist weder einzelne Erscheinung, die mit allen anderen im grenzenlosen Zusammenhang der Zeit und des Raumes unter dem Satz vom Grunde und dem ihm entspringenden Gesetz der Kausalität steht, noch der bloß diskursive, formelle und mechanische Begriff, sondern das, was Schopenhauer mit Platon „Idee“ nennt. Die Idee ist die ursprüngliche Einheit, die jeder Vielheit vorangeht und erst ,vermöge der Zeit-

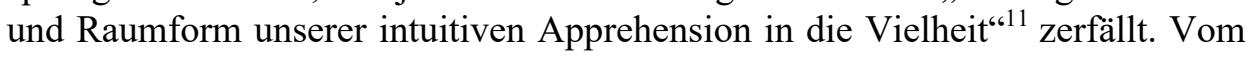
Begriff unterscheidet sich die Idee durch die ihr innewohnende höchste Lebendigkeit und d. h. die Fähigkeit, aus sich das Andere und Neue hervorzubringen:

[D]er Begriff gleicht einem toten Behältnis, in welchem, was man hineingelegt hat, wirklich nebeneinander liegt, aus welchen sich aber nicht mehr herausnehmen läßt (durch analytische Urteile), als man hinein gelegt hat (durch synthetische Reflexion); die Idee hingegen entwickelt in dem, welcher sie gefaßt hat, Vorstellungen, die in Hinsicht auf der ihr gleichnamigen Begriff neu sind: sie

\footnotetext{
${ }^{8}$ Dazu sei am Rande bemerkt, dass Schopenhauer, der sonst die ,intellektuellen Anschauung“ der sogen. Deutschen Idealisten unermüdlich kritisiert und unbedingt ablehnt, selbst - offensichtlich da er die Anschauung zunächst bloß formal, nämlich nur als eine intuitive und nicht diskursive Erkenntnis, bestimmt - ohne Vorbehalt von der ,im Verstande entspringende[n] Anschauung“ (Schopenhauer, „Die Welt als Wille und Vorstellung I,“ 420) sprechen zu dürfen meint.

${ }^{9}$ Schopenhauer, „Die Welt als Wille und Vorstellung I,“ 97.

${ }^{10}$ Ibid., 72.

${ }^{11}$ Ibid., 330.
} 
gleicht einem lebendigen, sich entwickelnden, mit Zeugungskraft begabten Organismus, welcher hervorbringt, was nicht in ihm eingeschachtelt liegt. ${ }^{12}$

Der Mensch als Subjekt der die Ideen anschauenden Erkenntnis wird von seiner Anschauung zutiefst berührt und unwiderstehlich mitgerissen. Die ganze Macht seines Geistes gibt sich der Anschauung hin, versenkt sich ganz in diese und verliert sich gänzlich in seinen Gegenstand. Indem er als Subjekt im angeschauten Gegenstand ganz aufgeht, ist er dieser Gegenstand selbst geworden ${ }^{13}:,[\mathrm{D}]$ as einzelne Objekt seiner Beschauung oder die übermäßig lebhaft von ihm aufgefaßte Gegenwart erscheinen in so hellem Licht, daß gleichsam die übrigen Glieder der Kette, zu der sie gehören, dadurch in Dunkel zurücktreten. " ${ }^{14}$ Für unseres Thema ist in all dem vor allem dies vom Belang, dass die intuitive Anschauung der Idee, die immer nur ,plötzlich“15 sich ereignet, eine solche Art der Erkenntnis ist, durch welche das anschauende bzw. erkennende Subjekt von Grund auf verändert und verwandelt wird. Die Kluft zwischen Subjekt und Objekt, die sonst als Grundvoraussetzung aller und jeder Erkenntnis gilt, ist hier aufgehoben. Das Subjekt geht in das Objekt auf, verliert sich in ihm, wird selbst mit dem Objekt eines und dasselbe, und findet aus dieser anschauenden Verschmelzung mit der Idee als ein wesentlich verändertes und gleichsam neugeborenes zu sich zurück. Das ist das Einzige, was als Erkenntnis im strengen Sinne gelten darf:

Die Anschauung [...] ist allein die unbedingt wahre, die echte, die ihres Namens vollkommen würdige Erkenntnis: denn sie allein erteilt eigentliche Einsicht, sie allein wird vom Menschen wirklich assimiliert, geht in sein Wesen über und kann mit vollem Grunde sein heißen; während die Begriffe ihm bloß ankleben. ${ }^{16}$

Die einzelne Erscheinung, die in sinnlichen Wahrnehmung und in der vernünftig begreifenden Erkenntnis nur als ein verschwindend kleiner Teil in dem Strome des Weltlaufs erscheint, wird dadurch, dass sie als Idee angeschaut und erkannt wird, plötzlich zu einem „Repräsentant des Ganzen, ein[em] Äquivalent des in Raum und Zeit unendlich Vielen." ${ }^{\text {"17 }}$ In Hinsicht darauf liegt es nahe, dem anschauenden Subjekt die wissende Vertrautheit mit dem Ganzen der Welt zuzumessen. Eben das tut Schopenhauer, der dem dergestalt erkennenden bzw. wissenden menschlichen Subjekt den Namen „Genie“ gibt und als dessen Tätigkeitsbereich die Kunst im weitesten Sinne bestimmt. Die im Bereich der Kunst sich ergebende Anschauung der Ideen sei die wahre und vollkommene Erkenntnis, solche nämlich, ,welche nicht auf einzelne Dinge gerichtet ist, sondern die

\footnotetext{
${ }^{12}$ Schopenhauer, „Die Welt als Wille und Vorstellung I,“ 330.

${ }^{13}$ Vgl. Schopenhauer, „Die Welt als Wille und Vorstellung I,“ 257. Auch Schopenhauer, „Die Welt als Wille und Vorstellung I,“ 259.

${ }^{14}$ Ibid., 277.

${ }^{15}$ Ibid., 256.

16 Ibid., 103.

${ }^{17}$ Ibid., 265.
} 
Ideen, also das ganze Wesen der Welt und des Lebens vollkommen aufgefaßt hat. ${ }^{\text {18 }}$ Sie sei die Quelle jeder anderen, auch der begrifflichen Erkenntnis. In diesem Zusammenhang stoßen wir auf eine wichtige Erwähnung der Weisheit: „Alle tiefe Erkenntnis, sogar die eigentliche Weisheit wurzelt in der anschaulichen Auffassung der Dinge [...]. Eine anschauliche Auffassung ist allemal der Zeugungsprozeß gewesen, in welchem jedes echte Kunstwerk, jeder unsterblicher Gedanke den Lebensfunken erhielt. Alles Urdenken geschieht in Bildern." ${ }^{\text {19 }}$

Das ist die erste ausdrückliche Bestimmung der Weisheit, wie sie von Schopenhauer verstanden wird. Demnach besteht die Weisheit in der intuitiven ${ }^{20}$ Anschauung der Ideen, in welche Anschauung das erkennende Subjekt völlig aufgeht, um mit dem Angeschauten zusammen zur lebendigen Quelle der Bilder zu werden, welche allem seinen Denken und Handeln zugrunde liegen. Dieses Moment des Praktischen kommt besonders stark zum Vorschein in der anderen Bestimmung der Weisheit, die wohl als eine Arte deren Definition zu nehmen ist: „,Weisheit ${ }^{\star}$ scheint mir nicht bloß theoretische, sondern auch praktische Vollkommenheit zu bezeichnen. Ich würde sie definieren als die vollendete, richtige Erkenntnis der Dinge im Ganzen und Allgemeinen, die den Menschen so völlig durchdrungen hat, daß sie nun auch in seinem Handeln hervortritt, indem sie sein Tun überall leitet. ${ }^{\text {“21 }}$ Es ist vor dem Hintergrund dieser stark betonten praktischen Bedeutung gewiss kein Zufall, dass Schopenhauer im selben Zusammenhang und gleichsam in einem Atem mit der Weisheit „die wahre Le-

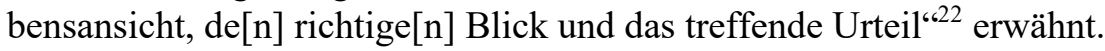

$* * *$

Rückt Schopenhauer mit dieser Weisheitsbestimmung, ohne es eigentlich zu wollen und auch zu ahnen, ganz nahe an den Begriff der Erfahrung, der auch sonst in der Philosophie, aber insbesondere bei seinem Erzfeind Hegel stark gemacht wird? Denn das Entscheidende an der Erfahrung, als welche der ganze dialektische Weg der Phänomenologie des Geistes bestimmt wird, liegt eben darin, dass das erfahrende Bewusstsein in seinen Gegenstand völlig aufgeht und

\footnotetext{
${ }^{18}$ Ibid., 327.

${ }^{19}$ Schopenhauer, „Die Welt als Wille und Vorstellung II,“ 488.

${ }^{20}$ Hier gilt es an die etymologischen Überlegungen von Karl Brugmann zu erinnern, der in seinem Aufsatz „Etymologische Miszellen“, Indogermanische Forschungen. Zeitschrift für indogermanische Sprach- und Altertumskunde 16 (1904): 491-509, insb. 499-503, der sprachlichen Sippe, zu der das griechische Wort бoøó $\varsigma$ gehört, die Grundbedeutung ,sehen“ beizumessen geneigt ist. In unserem Zusammenhang ist vor allem die Tatsache vom Belang, dass Brugmann den nächsten Verwandten von бофós mit seiner Sippe in lateinischem tuor findet, mit der Grundbedeutung ,ins Auge fassen, schauen, sich sorgend um etwas kümmern, wahren", woher dann über in-tuor der Name intuitio stamme.

${ }^{21}$ Schopenhauer, „Die Welt als Wille und Vorstellung V,“ 705.

${ }^{22}$ Schopenhauer, „Die Welt als Wille und Vorstellung I,“ 103.
} 
durch die Einsicht in die Endlichkeit bzw. die innere Grenze seiner jeweiligen Gestalt dazu gebracht wird, sich selbst zusammen mit diesem Gegenstand aufzugeben, um zur anderen, höheren Gestalt seiner selbst und des Gegenstandes hinaufzusteigen. Dem entsprechend besteht nach der Enzyklopädie das Prinzip der Erfahrung eben darin, „dass für das Annehmen und Für-Wahrhalten eines Inhalts der Mensch selbst dabei sein müsse. “23 Die Nähe und sogar eine gewisse Sinnesverwandtschaft dieser Bestimmungen der Erfahrung zu der Weisheitsauffassung Schopenhauers sind kaum zu übersehen.

Oder ist Schopenhauers Weisheitsauffassung doch eher als die Vorankündigung des Grundsatzes der philosophischen Hermeneutik zu verstehen, wie er etwa bei Gadamer ausgearbeitet wird? Bekanntlich hat Gadamer, und zwar in der entschiedenen Abweisung der bei Hegel vorherrschenden Tendenz, den Weg der Erfahrung in dem letzten, höchsten und absoluten Wissen sich vollenden zu lassen, mit Nachdruck darauf bestanden, dass die Erfahrung im wahren Sinne immer nur „die Einsicht in die Grenzen des Menschseins, die Einsicht in die Unaufhebbarkeit der Grenze zum Göttlichen hin“"24, und d. h. die „Erfahrung der eigenen Geschichtlichkeit", sein kann. ${ }^{25}$

Freilich scheinen manche Äußerungen Schopenhauers, die im Zusammenhang mit der Weisheit fallen, dafür zu sprechen, und zwar außer der schon erwähnten nicht ausdrücklichen, aber der Sache nach vollzogenen Gleichsetzung von Weisheit mit der wahren Lebensansicht, dem richtigen Blick und dem treffenden Urteil, vor allem die in diesem Zusammenhang des Öfteren erwähnten

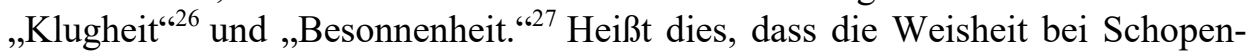
hauer, ähnlich wie die Erfahrung bei Gadamer, hauptsächlich in der Nachfolge

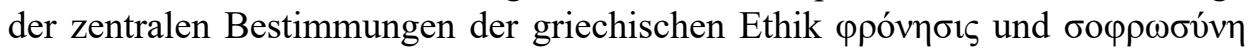
ins Auge gefasst und in mehr oder weniger bewussten Anknüpfung daran philosophisch ausgearbeitet wird?

Auch dafür gibt es Anzeichen. Bekanntlich ließ Gadamer seine Überlegungen zur Erfahrung als Einsicht des Menschen in eigene Endlichkeit und d. h. Geschichtlichkeit durch den Hinweis auf den alten griechischen Spruch $\pi \alpha ́ \theta \varepsilon \iota$

\footnotetext{
${ }^{23}$ Georg Wilhelm Friedrich Hegel, Enzylopädie der philosophischen Wissenschaften im Grundrisse (1830) I, $§ 7$ (G. W. F. Hegel, Werke in zwanzig Bänden. Theorie Werkausgabe. Auf der Grundlage der Werke von 1832-1842 neu edierte Ausgabe. Redaktion Eva Moldenhauer und Karl Markus Michel, Frankfurt am Main 1970), Bd. 8, S. 49 f.: „Das Prinzip der Erfahrung enthält die unendlich wichtige Bestimmung, daß für das Annehmen und Fürwahrhalten eines Inhalts der Mensch selbst dabei sein müsse, bestimmter, daß er solchen Inhalt mit der Gewissheit seiner selbst in Einigkeit und vereint finde. Er muß selbst dabei sein, sei es nur mit seinen äußerlichen Sinnen oder aber mit seinem tieferen Geist, seinen wesentlichen Selbstbewußtsein.“

${ }^{24}$ Hans-Georg Gadamer, Wahrheit und Methode. Grundzüge einer philosophischen Hermeneutik (H.-G. Gadamer, Gesammelte Werke, Bd. 1, Tübingen 1990), 363.

${ }^{25}$ Ibid.

${ }^{26}$ Schopenhauer, „Die Welt als Wille und Vorstellung I,“ 271.

${ }^{27}$ Ibid., 420.
} 
$\mu \alpha$ ó $\theta$ o bekräftigen, der etwa „durch Leiden belehrt“ heißt. Er greift zurück zum Agamemnon des Aischylos, wo der höchste Gott Zeus vom Chor als jener gepriesen wird, der die Sterblichen auf den Weg der Weisheit bringt und dem Spruch „durch Leid gelehrt“ seine volle Geltung verleiht. ${ }^{28}$ An einer anderen Stelle desselben Dramas wird der Spruch vom Chor wieder erwähnt, und zwar bei der Schilderung der Macht und Tatkraft der Dike, der Göttin der Gerechtigkeit, die „,demjenigen, der Leid duldet, auch Lernen noch dazu wägt.“29

Gadamer hebt hervor, dass durch das hier gemeinte Leiden der Mensch nicht dieses oder jenes lernen, sondern vor allem die Grenzen des Menschseins einsehen soll. Der Spruch bezieht sich auf die wesentliche Erfahrung des Menschen, welche keine andere ist als die Erfahrung der menschlichen Endlichkeit: „Erfahren im eigentlichen Sinne ist, wer ihrer inne ist, wer weiß, dass er der Zeit und der Zukunft nicht Herr ist. “30

Zweifellsohne stimmen diese Überlegungen Gadamers mit dem überein, was Aischylos durch den Spruch sagen wollte. Die durch das Leid - das bei den Griechen den ganzen weiten Spektrum von unmittelbaren physischen Schmerz, wie z. B. bei Io, über die unüberwindliche Beleidigung der persönlichen Würde, wie bei Aias etwa, bis zum rein geistigen Leiden am Fehlen des entsprechenden Wissens um das geheimnisvolle Rätsel des Sphinx zu lösen, wie bei Ödipus, in sich schließt - zu erlangende Weisheit ist ihrer Wesen nach kein theoretisches Wissen, sondern die entscheidende Einsicht des Menschen in das, was für sein Wesen als einen Sterblichen allein förderlich und dieses Wesen allein bewahrend ist. Nur derjenige, der über ein solches Wissen verfügt, verdient, als „der weise" im eigentlichen Sinne bezeichnet zu werden. Aischylos spricht davon überall in seinem Werk, ganz eindeutig in einem Fragment, das lautet: ó $\chi \rho \eta ́ \sigma \mu$

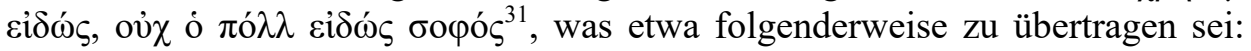
„Weise ist [nur] jener, der das Wissen vom für den Menschen Förderlichen, d. h. von dem, was zur gelingenden Lebensführung des Menschen entscheidend ist, hat, und nicht von Vielem.“

Es leuchtet ein, inwiefern Aischylos in Hinsicht auf das menschliche Wissen, wie es gewöhnlich verstanden wird, vom Grund auf vorsichtig, wenn nicht sogar skeptisch ist. Wie nicht nur aus dem Gebundenen Prometheus ersichtlich, ist die unaufhaltsam forschende Wissenschaft in seinen Augen ,eine stete Gefährdung

\footnotetext{
${ }^{28}$ Aischylos. Agamenon, in Aeschylus, Agamemnon, Libation-Bearers, Eumenides, Fragments, Band 2, with an English translation by Herbert Weir Smyth (Cambridge, Mass.: Harvard University

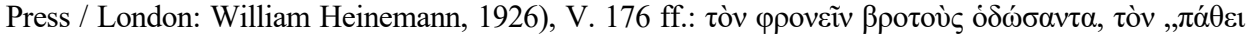

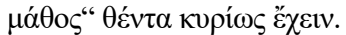

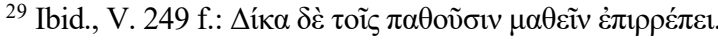

${ }^{30}$ Gadamer, Wahrheit und Methode, 363.

31 Aischylos, Fragments, in Aeschylus, Agamemnon, Libation-Bearers, Eumenides, Fragments, Band 2, with an English translation by Herbert Weir Smyth (Cambridge, Mass.: Harvard University Press / London: William Heinemann, 1926), $390 \mathrm{~N}^{2}$ (667 M).
} 
und Last für den Menschen. “32 Der Grund dafür liegt darin, dass das Wissen bei den Menschen stets der Gefahr der Überheblichkeit und der verhängnisvollen Nichtbeachtung der Kluft, die das vollkommene Wissen der Götter von dem dem Menschen einzig zugemessenen flüchtigen und unbeständigen Dafürhalten trennt, ausgesetzt bleibt. Die einzige Form des Wissens, die Aischylos anerkennt, ist ,das $\sigma \omega \varphi \rho o v \varepsilon i v$, die Einsicht, die aus dem Handeln und Leiden erwächst und sich im Einklang mit der waltenden Ordnung weiß.“33

Auch bei Heraklit, dessen geistige Nähe zum Aischylos längst erkennt ist und der nicht weniger als jener das Wissen verstanden als bloßes „Vielwissen“ ( $\pi \circ \lambda v \mu \alpha \theta i ́ \eta)$ schlicht ablehnt, gehören die Verben $\varphi \rho \circ v \varepsilon \tilde{v}$ und $\sigma \omega \varphi \rho \circ v \varepsilon \tilde{v}$ zu

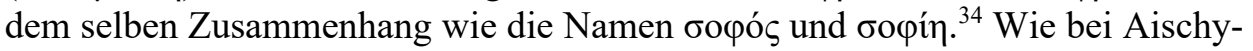
los im Agamemnon die eigentliche, durch das Leid erworbene Weisheit den Menschen zur freien Einfügung in die höhere, von Zeus bzw. Dike hergestellte und stets bewahrte Ordnung des Ganzen bringen soll, bedeutet бopín auch sonst bei den archaischen griechischen Dichtern und Denkern ,immer ein Wissen, welches das einzelne Handeln unter eine größere Ordnung stellt", des Näheren „ein Wissen, das die Stellung des Einzelnen im Ganzen erkennt und es bewußt in diese Ordnung einfügt. “35

In allen Fällen, wo bei den frühgriechischen Autoren eine Wendung aus dem

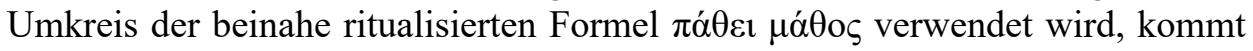
ein und derselbe Sachverhalt zum Vorschein, der in einer klassischen diesbezüglichen Studie folgenderweise zusammenfasst wurde: „Schmerz und Qual, Not und Strafe sind das letzte Mittel, durch das die Gottheit den Menschen gewaltsam korrigiert; gegen seinen Willen bringt sie ihn zur Anerkennung dieser Weltordnung und ihrer Gesetze. ${ }^{\text {" }}{ }^{36}$ Insofern ist es trotz dem unbestreitbaren darin liegenden Anachronismus letztendlich doch nicht unangemessen, in diesem $\mathrm{Zu}-$ sammenhang von ,eine[r] Art nicht doktrinaler, sondern in der Tragödienform performativ-ästhetische[r] Theodicee ${ }^{\text {(37 } 37} \mathrm{zu}$ sprechen.

Im Allgemeinen entspricht dies der wechselnden und stufenweise sich entfaltenden Grundbedeutung vom „Weisen“ (бoфós), wie sie etwa in der Zeitspanne von Homer bis Platon sich festgelegt hat. Zunächst wird durch dieses Wort ein jeder bezeichnet, der sich auf ein Handwerk meisterhaft versteht. Diese Bedeutung wird aber schnell erweitert und auf den verständigen Künstler und Dichter

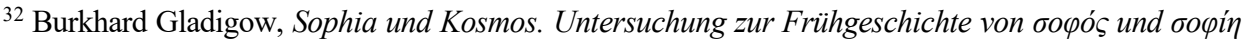
(Hildesheim: Georg Olms Verlag, 1965), 132.

${ }^{33}$ Ibid. Dem ist hinzusetzen, dass bezeichnenderweise an der ersten der von uns angegeben Stellen aus Agamemnon das, was sonst „Weisheit“ heißt, nicht mit durch den Namen бo甲ín, sondern durch

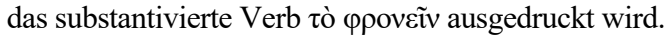

${ }^{34}$ Ibid., 114.

${ }^{35}$ Ibid., 74.

${ }^{36}$ Heinrich Dörrie, Leid und Erfahrung. Die Wort- und Sinn-Verbindung $\pi \alpha \theta \varepsilon \tilde{v}-\mu \alpha \theta \varepsilon \tilde{\imath} v$ im griechischen Denken (Wiesbaden: Steiner, 1956), 38.

${ }^{37}$ Philipp Stoellger, Passivität aus Passion (Tübingen: Mohr Siebeck, 2010), 32.
} 
verlagert, wodurch zur beträchtlichen Bedeutungserhöhung in Richtung auf das Geistige kam. Wie es vor allem bei Pindar ofenkundig wird, nimmt jetzt der Dichter für sich in Anspruch, der wahre бoфós zu sein, da er aufgrund seiner Vertrautheit mit den Musen, und damit auch mit der ganzen Sphäre des Göttlichen, für jedes, was sich in menschlichem Bereich abspielt, den rechten und ihm gebührenden Ort im Ganzen des Seienden zeigen kann. Aufgrund dessen wird in einem weiteren Schritt die Weisheit (бoфín) schon ganz allgemein auf das Erkennen und Verstehen der göttlichen Ordnung übertragen, was wohl am ausdrücklichsten bei Solon und Heraklit zum Vorschein kommt.

Allen diesen Stufen des Bedeutungswandels bleibt aber eines gemeinsam, dass nämlich die Weisheit ,nie bloße Erkenntnis oder die geistige Haltung eines dem Handeln entfremdeten Denkers ${ }^{638}$ ist, sondern immer in einer Wirksamkeit erscheint, welche des Näheren darin besteht, den Menschen das, was im Rahmen des Ganzen der menschlichen und göttlichen Welt und in Hinsicht darauf das wirklich Wert- und Würdevolle ist, vor Augen zu bringen. Erst vor diesem Hintergrund könnte, nebenbei gesagt, Nietzsches zunächst befremdliche Erklärung der frühgriechischen Weisheit aufleuchten, die er zur Zeit seiner philologischen Tätigkeit in Basel gibt: „,oøía bezeichnet das Wählende, mit Geschmack Ausscheidende: während sich die Wissenschaft ohne solchen Feingeschmack auf alles Wissbare stürzt.“39

Werfen wir abschließend noch einen ganz flüchtigen Blick auf Schopenhauer. Ist seine Weisheitsauffassung als der späte Nachklang der durch das Leid zu erlangenden frühgriechischen бoфín zu verstehen? In seinem Werk gibt es manches, was eine bejahende Antwort naheliegend macht. So sieht er z. B. die wesentliche Wirkung des Trauerspiels darin, den Menschen durch den „namenlose[n] Schmerz" durchgehen zu lassen, bis in ihm die Erkenntnis, ,geläutert und gesteigert durch das Leiden selbst, den Punkt erreicht, wo die Erscheinung, der Schleier der Maja, sie nicht mehr täuscht ${ }^{\star 40}$, woher ihr dann die endgültige Einsicht in die unaufhebbare Nichtigkeit des Willens zum Leben entspringen soll. Nach Schopenhauers Ansicht beruht auch sonst die eigentümliche Wirkung des Trauerspiels ,im Grunde darauf, daß es jenen angeborenen Irrtum erschüttert, indem es die Vereitelung des menschlichen Strebens und die Nichtigkeit dieses ganzen Daseins an einem großen und frappanten Beispiel lebhaft veranschau-

\footnotetext{
${ }^{38}$ Gladigow, Sophia und Kosmos, 73. Der oben gegebene Umriss der Bedeutungsentwicklung von бофó

${ }^{39}$ Nietzsche's Werke, hg. von O. Crusius und Wilhelm Nestle, Bd. 19, Philologica 3: Unveröffentliches zur antiken Religion und Philosophie (Leipzig: Alfred Kröner, 1913), 134.

${ }^{40}$ Schopenhauer, „Die Welt als Wille und Vorstellung I,“ 353.
} 
licht und hiedurch den tiefsten Sinn des Lebens aufschließt.“" ${ }^{41}$ In einer nicht zu übersehenden Übereinstimmung mit dem Wesen der griechischen Tragödie ist auch für Schopenhauer das Leiden - zu welchem allgemeinen Begriff auch für ihn sowohl der körperliche als auch der „Geistesschmerz"“42 sowie das „heftige[...] geistige[...] Leiden“, vor allem aber das unheimlichste und kaum zu ertragende Leiden an der Leere der Zeit, die Langeweile, gehören - ,in der Tat der Läuterungsproze $3^{\text {‘43 }}$, durch welchen dem Menschen die vollkommene Erkenntnis des tiefsten Wesens und der Wahrheit des Lebens und der Welt entspringt.

Jedoch gilt es darauf zu bestehen, dass eine ganze Welt zwischen der aus diesem Läuterungsprozess hervorkommenden Weisheit und der alten griechischen бo甲í sich schiebt. Hier schränken wir uns zunächst auf den Hinweis darauf ein, dass Schopenhauer die Weisheit nicht dabei belässt, bloß als praktische Vollkommenheit das Tun des Menschen zu leiten, wie sie in Parerga und Paralipomena anscheinend maßgeblich bestimmt wird. Wie der Einleitung zu den darin enthaltenen Aphorismen zur Lebensweisheit zu entnehmen ist, die Lebensweisheit verstanden im Sinne ,der Kunst, das Leben möglichst angenehm und glücklich durchzuführen“ bzw. der „Anweisung zu einem glücklichen Dasein" erkennt Schopenhauer nicht als seine eigene Sache an. Entschieden und unmissverständlich erklärt er, dass seine Philosophie die Frage ob ,das menschliche Leben dem Begriff eines solchen Daseins entspreche oder auch nur entsprechen könne" schlicht verneint und zum Zweck der Ausarbeitung der Lebensweisheit „von dem höheren metaphysisch-ethischen Standpunkte, zu welchem [s]eine eigentliche Philosophie hinleitet [...] gänzlich abgehn" musste. ${ }^{44}$

Die Weisheit, wie er sie eigentlich versteht, begnügt sich nicht damit, bloße Besonnenheit und Nüchternheit zu sein. Obwohl die von ihm entworfene Lebensweisheit in manchen Grundzügen an die überlieferte stoische Ethik angelehnt ist, das stoische Ideal des allem Leiden enthobenen und daher glückseligen Weisen befriedigt ihn nicht:

$\mathrm{Da}$ aber, nach dem stoischen Plan, etwas Vollkommnes zu Stande komme, wirklich die richtig gebrauchte Vernunft uns aller Leiden enthöbe und zum glückseligen Weisen mache; daran fehlt sehr viel. Es liegt vielmehr [ein] vollkommner Widerspruch darin, Leben zu wollen ohne zu leiden, den auch das oft

\footnotetext{
${ }^{41}$ Schopenhauer, „Die Welt als Wille und Vorstellung I“, 815. Bekannterweise hat das Leiden bei Schopenhauer nich bloß eine physische oder auch psychische, sondern in erster Linie eine metaphysische, und d. h. universelle Bedeutung. Vgl. dazu Oliver Hallich, „Ethik“, in Daniel Schübbe/Mathias Koßler (Hg.), Schopenhauer Handbuch. Leben - Werk - Wirkung, 2. aktualisierte und erweiterte Auflage (Stuttgart: J. B. Metzler, 2018), 83: „Schopenhauers Ethik ist eine Leidensethik in dem Sinne, dass sie die Welt als Leidensgeschehen auffasst. Ebenso wie die Welt auch dort Wille ist, wo keinerlei Intentionalitćt oder Bewusstsein vorliegt, ist das Dasein auch dort Leiden, wo dieses nicht als solches empfunden wird.“

${ }^{42}$ Schopenhauer, „Die Welt als Wille und Vorstellung I,“ 420.

${ }^{43}$ Schopenhauer, „Die Welt als Wille und Vorstellung II,“ 816.

${ }^{44}$ Schopenhauer, „Die Welt als Wille und Vorstellung I,“ 375.
} 
gebrauchte Wort ,seliges Leben“ an sich trägt. [...] Dieser Widerspruch tritt schon hervor an dem aufgestellten Ideal des Weisen, der in ihrer Darstellung niemals Leben oder innere poetische Wahrheit gewinnen konnte; sondern oft zum leblosen Stein wird, immer aber ein hölzerner steifer Gliedermann bleibt, mit dem nichts anzufangen ist, der selbst nicht weiß wohin mit seiner Weisheit, dessen vollkommne Ruhe, Zufriedenheit und Glückseligkeit dem Wesen des Menschen geradezu widerspricht und uns zu keiner anschaulichen Vorstellung davon kommen lässt. ${ }^{45}$

Dem Genie spricht Schopenhauer die Nüchternheit ausdrücklich ab. ${ }^{46}$ Die ihm eigentümliche echte Weisheit führt ihn über alles praktische Handeln und theoretische Wissen weit hinaus. Insofern ist sie verschieden auch davon, was in der Philosophie „Erfahrung“ heißt, abgesehen davon ob diese wie bei Hegel etwa als der Weg zum absoluten Wissen oder wie bei Gadamer als die Einsicht in die unaufhebbare Endlichkeit des Menschen verstanden wird. Von all dem trennt Schopenhauer die Weisheit, wie er sie verstehen will, indem er sie emphatisch als ,tiefe“" und ,letzte“"Weisheit bezeichnet. Sie hält nicht an bevor sie den Menschen zum letzen Gipfel bringt, dorthin wo sie - seiner Überzeugung nach im vollen Einklang mit dem innersten Geist des Christentums und der uralten indischen Weisheit, freilich wie er beides versteht - sich als ,,aus der läuternden Flamme des Leidens plötzlich hervortretende Silberblick der Verneinung des Willens zum Leben, d. h. der Erlösung ${ }^{647}$ zeigt. Durch das äußerste, zur Weisheit führende Leid soll der Mensch also nicht bloß belehrt, sondern darüber hinaus ,geheiligt, d. h. von dem Irrweg des Willens zum Leben zurückgeführt“، werden. $^{48}$

Um den kaum zu ermessenden Unterschied, durch welchen solcherweise verstandene Weisheit geschieden bleibt von der erhabenen Würde jener griechischen, die auch im Angesicht des höchsten Leides den nie auszutilgenden Glanz und die reizende Anmut des Lebens zu bejahen vermochte, wirklich zu erkennen, wäre es nötig, das Thema noch einmal von vorne anzugreifen, und zwar viel eingehender, nämlich so, dass dabei der Wille als die zentrale und alles entscheidende Sache der Philosophie Schopenhauers von Anfang an einbezogen würde. Dies bleibe einem nächsten Schritt überlassen.

\footnotetext{
${ }^{45}$ Arthur Schopenhauer, Theorie des gesammten Vorstellens, Denkens und Erkennens. Aus dem handschriftlichen Nachlass herausgegeben und eingeleitet von Volker Sperling (München/Zürich: Piper, 1986), 440.

${ }^{46}$ Schopenhauer, „Die Welt als Wille und Vorstellung I,“ 277.

${ }^{47}$ Ibid., 533.

${ }^{48}$ Schopenhauer, „Die Welt als Wille und Vorstellung II,“ 816.
} 


\section{BIBLIOGRAPHIE}

Aischylos. Agamemnon. In: Aeschylus, Agamemnon, Libation-Bearers, Eumenides, Fragments, Band 2, with an English translation by Herbert Weir Smyth. Cambridge, Mass.: Harvard University Press / London: William Heinemann, 1926.

- Fragments. In: Aeschylus, Agamemnon, Libation-Bearers, Eumenides, Fragments, Band 2, with an English translation by Herbert Weir Smyth. Cambridge, Mass.: Harvard University Press / London: William Heinemann, 1926.

Brugmann, Karl. „Etymologische Miszellen“, Indogermanische Forschungen. Zeitschrift für indogermanische Sprach- und Altertumskunde, Nr. 16 (1904): 491-509.

Dörrie, Heinrich. Leid und Erfahrung. Die Wort- und Sinn-Verbindung $\pi \alpha \theta \varepsilon \tilde{\imath} v-\mu \alpha \theta \varepsilon \tilde{v} v$ im griechischen Denken. Wiesbaden: Steiner, 1956.

Gadamer, Hans-Georg. Wahrheit und Methode. Grundzüge einer philosophischen Wahrheit und Methode. Grundzüge einer philosophischen Hermeneutik. Tübingen: Mohr Siebeck, 1990.

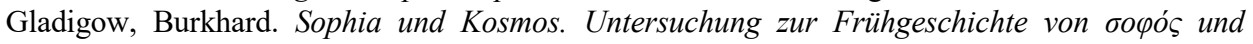
бoøí. Hildesheim: Georg Olms Verlag, 1965.

Gloy, Karen. Von der Weisheit zur Wissenschaft. Freiburg/München: Verlag Karl Alber, 2007.

Hallich, Oliver. „Ethik“. In: Daniel Schübbe/Mathias Koßler (Hg.), Schopenhauer Handbuch. Leben - Werk - Wirkung, 2. aktualisierte und erweiterte Auflage. Stuttgart: J. B. Metzler, 2018.

Hegel, Georg Wilhelm Friedrich. Enzyklopädie der philosophischen Wissenschaften im Grundrisse (1830) I. In: Georg Wilhelm Friedrich Hegel, Werke in zwanzig Bänden. Theorie Werkausgabe. Auf der Grundlage der Werke von 1832-1842 neu edierte Ausgabe. Redaktion Eva Moldenhauer und Karl Markus Michel, Frankfurt am Main: Suhrkamp, 1970.

Nietzsche's Werke, hg. von Otto Crusius und Wilhelm Nestle, Bd. 19, Philologica 3: Unveröffentliches zur antiken Religion und Philosophie. Leipzig: Alfred Kröner, 1913.

Schopenhauer, Arthur. Sämtliche Werke. Textkritisch bearbeitet und herausgegeben von Wolfgang Frhr. von Löhneysen. Frankfurt am Main: Wissenschaftliche Buchgesellschaft Darmstadt, 1968.

Schopenhauer, Arthur. Theorie des gesammten Vorstellens, Denkens und Erkennens. Aus dem handschriftlichen Nachlass herausgegeben und eingeleitet von Volker Sperling. München/Zürich: Piper, 1986.

Stoellger, Philipp. Passivität aus Passion. Tübingen: Mohr Siebeck, 2010.

\section{PATHEI MATHOS. \\ SCHOPENHAUER'S DOCTRINE OF WISDOM AND THE ANCIENT GREEK SOPHIA}

In the face of growing de-objectification and depersonalization, which in the realm of knowledge have asserted themselves through the unconditional claim of science to objectivity, it is advisable to remember the old, and for the most part now forgotten, concept of wisdom, and its philosophical scope. This is here done using Schopenhauer as an example. In his philosophy, wisdom plays a central role, as a not only theoretical but also practical perfection of man. In order to grasp the essence of the wisdom so highly placed as accurately as possible, it is confronted in the essay, on the one hand, with Gadamer's concept of hermeneutic experience and, on the other hand, with the concept of wisdom expressed in the ancient Greek saying "taught by suffering". The investigation leads to the conclusion that the wisdom by Schopenhauer, although at first sight similar and in part even related to the two concepts of wisdom mentioned above, is however fundamentally different from them, since its purpose is not to instruct man about the insurmountable limits of his mortality and thereby assign to him his appointed place in life, but rather to lead him away from the will to live at all.

Keywords:

Schopenhauer, wisdom, will, knowledge, pain, suffering 